\title{
Erratum to: Fragment-based in silico modeling of multi-target inhibitors against breast cancer-related proteins
}

\author{
Alejandro Speck-Planche ${ }^{1}$ (I) - M. Natália D. S. Cordeiro ${ }^{1}$
}

Published online: 1 August 2017

(C) Springer International Publishing AG 2017

\section{Erratum to: Mol Divers}

\section{DOI 10.1007/s11030-017-9731-1}

In the original publication, the equations were published incorrectly. The corrected equations are given below.

$$
\begin{aligned}
& q_{k}(\bar{x})=\left[X^{T}\right][\mathrm{M}]^{k}[X]=\sum_{i=1}^{n} \sum_{j=1}^{n}{ }^{k} m_{i j} \cdot x_{i} \cdot x_{j} \\
& M q_{k}(P P)=\frac{q_{k}(P P)}{n A T} \\
& \operatorname{avg\_ } M q_{k}(P P) c_{j}=\frac{1}{n\left(c_{j}\right)} \sum_{i=1}^{n\left(c_{j}\right)} M q_{k}(P P)_{i}
\end{aligned}
$$

$$
D M q_{k}(P P)_{i} c_{j}=G M p \cdot\left[M q_{k}(P P)_{i}-a v g_{-} M q_{k}(P P) c_{j}\right]
$$

$$
\begin{aligned}
\operatorname{SACT}_{i}\left(c_{j}\right)= & a_{0}+\sum_{m=1}^{z} b_{m} \cdot\left[D M q_{k}(P P)_{i} c_{j}\right]_{m} \\
\operatorname{SACT}_{i}\left(c_{j}\right)= & 0.33 D M q_{5}(H Y D) m_{a}+0.12 D M q_{3}(A W) m_{a} \\
& +14.33 D M q_{0}(H Y D) b_{t}-0.03 D M q_{0}(P S A) b_{t} \\
& -0.25 D M q_{1}(A R) a_{i}+1.45 \\
N=18,230, & \lambda=0.4, \chi^{2}=16,678, p \text { value }<10^{-16}
\end{aligned}
$$

The online version of the original article can be found under doi:10.1007/s11030-017-9731-1.

\section{Alejandro Speck-Planche} alejspivanovich@gmail.com

$\bowtie$ M. Natália D. S. Cordeiro ncordeir@fc.up.pt

1 LAQV@REQUIMTE/Department of Chemistry and Biochemistry, University of Porto, 4169-007 Porto, Portugal 